\title{
On reciprocality of twisted Alexander invariants
}

\author{
JONATHAN A HILLMAN \\ DANIEL S SILVER \\ SUSAN G WiLliams
}

\begin{abstract}
Given a knot and an $\mathrm{SL}_{n} \mathbb{F}$ representation of its group that is conjugate to its dual, the representation that replaces each matrix with its inverse-transpose, the associated twisted Reidemeister torsion is reciprocal. An example is given of a knot group and $\mathrm{SL}_{3} \mathbb{Z}$ representation for which the twisted Reidemeister torsion is not reciprocal.
\end{abstract}

$57 \mathrm{M} 25$

\section{Introduction}

Let $R$ be a ring. A Laurent polynomial $f(t) \in R\left[t^{ \pm 1}\right]$ is reciprocal if $f(t)=u f\left(t^{-1}\right)$, for some unit $u \in R\left[t^{ \pm 1}\right]$. If $R$ has no zero divisors, the condition is equivalent to $f\left(t^{-1}\right)= \pm t^{i} f(t)$, for some $i \in \mathbb{Z}$.

The Alexander polynomial $\Delta(t)$ of a knot $k$ can be computed from a diagram of $k$ or from a presentation of the knot group (see Kawauchi [5], for example). It is an integral Laurent polynomial, well defined up to multiplication by units, and usually normalized to be a polynomial with nonzero constant coefficient. It is well known that $\Delta(t)$ is reciprocal. This is a consequence of Poincaré duality of the knot exterior (see Torres and Fox [15] for an alternative approach based on duality in the knot group).

In 1990 X S Lin introduced a more sensitive knot invariant by using information from representations of the knot group [10]. Later, refinements were described by M Wada [16] and others including P Kirk and C Livingston [6], and J Cha [1]. These twisted Alexander invariants have proven to be useful for a variety of questions about knots including questions about concordance [6], knot symmetry (see Hillman, Livingston and Naik [4]), and fibrations (see Friedl and Vidussi [2]). See Friedl and Vidussi [3] for a survey.

We briefly review the definition of perhaps the best-known twisted Alexander invariant. Let $k$ be a knot with exterior $X$, endowed with the structure of a CW complex. We fix a Wirtinger presentation $\left\langle x_{0}, x_{1}, \ldots, x_{k} \mid r_{1}, \ldots, r_{k}\right\rangle$ for the knot group $\pi=\pi_{1}(X)$. 
Let $\phi: F_{k} \rightarrow \pi$ be the associated projection of the free group $F_{k}=\left\langle x_{0}, x_{1}, \ldots, x_{k} \mid\right\rangle$ to $\pi$. It induces a ring homomorphism $\tilde{\phi}: \mathbb{Z}\left[F_{k}\right] \rightarrow \mathbb{Z}[\pi]$.

Let $\epsilon: \pi \rightarrow H_{1}(X ; \mathbb{Z}) \cong\langle t \mid\rangle$ be the abelianization mapping each $x_{i}$ to $t$. It induces a ring homomorphism $\tilde{\epsilon}: \mathbb{Z}[\pi] \rightarrow \mathbb{Z}\left[t^{ \pm 1}\right]$.

Let $R$ be a Noetherian unique factorization domain. Assume that $\gamma: \pi \rightarrow \mathrm{GL}_{n} R$ is a linear representation. Let $\tilde{\gamma}: \mathbb{Z}[\pi] \rightarrow M_{n}(R)$ be the associated ring homomorphism to the algebra of $n \times n$ matrices over $R$. We obtain a homomorphism

$$
\tilde{\gamma} \otimes \tilde{\epsilon}: \mathbb{Z}[\pi] \rightarrow M_{n}\left(R\left[t^{ \pm 1}\right]\right),
$$

mapping $g$ to $\epsilon(g) \gamma(g)$, that we denote more simply by $\Phi$.

Let $M_{\gamma \otimes \epsilon}$ denote the $k \times(k+1)$ matrix with $(i, j)$-component equal to the $n \times n$ matrix $\Phi\left(\frac{\partial r_{i}}{\partial x_{j}}\right) \in M_{n}\left(R\left[t^{ \pm 1}\right]\right)$. Here $\frac{\partial r_{i}}{\partial x_{j}}$ denotes Fox partial derivative. Let $M_{\gamma \otimes \epsilon}^{0}$ denote the $k \times k$ matrix obtained by deleting the column corresponding to $x_{0}$. We regard $M_{\gamma \otimes \epsilon}^{0}$ as a $k n \times k n$ matrix with coefficients in $R\left[t^{ \pm 1}\right]$.

Definition 1.1 The Wada invariant $W_{\gamma}(t)$ is

$$
\frac{\operatorname{det} M_{\gamma \otimes \epsilon}^{0}}{\operatorname{det} \Phi\left(x_{0}-1\right)} \text {. }
$$

When $\gamma$ is the trivial 1-dimensional representation, $M_{\gamma \otimes \epsilon}^{0}$ is a matrix $M(t)$ that we call the Alexander matrix of $k$. (This terminology is used, for example, by Rolfsen [13], but it is not standard.) The determinant of $M(t)$ is the (untwisted) Alexander polynomial $\Delta(t)$ of $k$.

Remark 1.2 Although the rational function $W_{\gamma}(t)$ is often a polynomial, it need not be. However, in general it is well defined up to multiplication by $(-t)^{n i}$. See Wada [16].

Let $\tilde{X}$ denote the universal cover of $X$, with the structure of a CW complex that is lifted from $X$. The matrix $M_{\gamma \otimes \epsilon}$ represents a boundary homomorphism for a twisted chain complex

$$
C_{*}\left(X ; V\left[t^{ \pm 1}\right]_{\gamma}\right)=\left(R\left[t^{ \pm 1}\right] \otimes_{R} V\right) \otimes_{\gamma} C_{*}(\tilde{X}) .
$$

Here $V=R^{n}$ is a free module on which $\pi$ acts via $\gamma$, while $C_{*}(\tilde{X})$ denotes the cellular chain complex of $\tilde{X}$ with coefficients in $R$. The group ring $R[\pi]$ acts on the 
left via deck transformations. On the other hand, $R\left[t^{ \pm 1}\right] \otimes_{R} V$ has the structure of of a right $R[\pi]$-module via

$$
(p \otimes v) \cdot g=(\epsilon(g) p) \otimes(v \gamma(g)), \text { for } \gamma \in \pi .
$$

The homology group $H_{1}\left(X ; V\left[t^{ \pm 1}\right]\right)$ of the chain complex (1-2) is a finitely generated $R\left[t^{ \pm 1}\right]$-module. Its 0th elementary divisor $\Delta_{\gamma}(t)$, which is well defined up to multiplication by units in $R\left[t^{ \pm 1}\right]$, lately competes with $W_{\gamma}(t)$ for the name "twisted Alexander polynomial." In many cases they are equal (up to multiplication by units); generally, $\Delta_{\gamma}(t)$ is $\operatorname{det} M_{\gamma \otimes \epsilon}^{0}$ divided by a factor of $\operatorname{det} \Phi\left(x_{0}-1\right)$. See Kirk and Livingston [6] or Silver and Williams [14] for details.

The representation $\gamma$ induces a representation $\gamma: \pi \rightarrow G L_{n}(\mathbb{F}(t))$, where $\mathbb{F}(t)$ is the field of fractions of $R\left[t^{ \pm 1}\right]$. When $\operatorname{det} M_{\gamma \otimes \epsilon}^{0} \neq 0$, the chain complex

$$
\left.C_{*}\left(X ; V(t)_{\gamma}\right)=(\mathbb{F}(t)) \otimes_{R} V\right) \otimes_{\gamma} C_{*}(\tilde{X})
$$

is acyclic (see Kitano [7]), and hence the Reidemeister torsion $\tau_{\gamma}(t)$ is defined. It coincides with the Wada invariant (see [7] and also [6]).

Remark 1.3 (1) Conjugating the representation $\gamma$ corresponds to a change of basis for $V$. It is well known that the invariants $\Delta_{\gamma}(t)$ and $\tau_{\gamma}(t)$ are unchanged.

(2) The indeterminacy of sign in the definition of $\tau_{\gamma}(t)$ can be removed (see Kitayama [8]).

T Kitano used Poincaré duality to prove in [7] that for orthogonal representations $\gamma: \pi \rightarrow \mathrm{SO}_{n}(\mathbb{R})$, the torsion $\tau_{\gamma}(t)$ is reciprocal, where reciprocality for rational functions is defined as for Laurent polynomials. (In fact Kitano shows that $\tau_{\gamma}\left(t^{-1}\right)$ and $\tau_{\gamma}(t)$ are equal up to multiplication by $\pm t^{n i}$.) He asked whether reciprocality holds for more general representations.

For representations $\gamma: \pi \rightarrow \mathrm{GL}_{n}(\mathbb{C})$, "reciprocality" can have another meaning. One can require that $\tau_{\gamma}(t)$ be equal up to multiplication by a unit to the expression obtained by inverting $t$ and also taking complex conjugates of coefficients. Kirk and Livingston showed in [6] that $\tau_{\gamma}(t)$ satisfies such a condition whenever $\gamma$ is unitary.

It is not difficult to find representations $\gamma: \pi \rightarrow \mathrm{GL}_{n} \mathbb{F}$ such that $\tau_{\gamma}(t)$ is non-reciprocal. For example, consider the Wirtinger presentation

$$
\left\langle x_{0}, x_{1}, x_{2} \mid x_{0} x_{1}=x_{2} x_{0}, x_{1} x_{2}=x_{0} x_{1}\right\rangle
$$


of the trefoil knot group $\pi$. The assignment $x_{i} \mapsto X_{i} \in \mathrm{GL}_{1} \mathbb{F}$, such that $X_{i}=(2), i=$ $0,1,2$, yields the non-reciprocal invariant

$$
\tau_{\gamma}(t)=\frac{4 t^{2}-2 t+1}{2 t-1} .
$$

(This simple example was suggested to us by S Friedl.) The question of reciprocality for representations in $\mathrm{SL}_{n} \mathbb{F}$ is more subtle. The question was proposed by Kitano [7]; it appeared recently in [3].

In Section 2 we show that reciprocality need not hold for general representations in $\mathrm{SL}_{n} \mathbb{F}$. The representations $\gamma$ that we consider have the property that the dual representation $\bar{\gamma}$, obtained by replacing each matrix $\gamma(g), g \in \pi$, by its inversetranspose, is not conjugate to $\gamma$.

In Section 3 we prove that if a representation $\gamma: \pi \rightarrow \mathrm{GL}_{n} \mathbb{F}$ is conjugate to its dual, then the torsion $\tau_{\gamma}(t)$ is reciprocal.

\section{Examples}

Any reciprocal even-degree integral polynomial $\Delta(t)$ such that $\Delta(1)= \pm 1$ arises as the Alexander polynomial of a knot (see Kawauchi [5], for example). Let $f(t)$ be any monic integral polynomial with constant coefficient -1 and $f(1)= \pm 1$. Choose a knot $k$ with Alexander polynomial $\Delta(t)=f(t) f\left(t^{-1}\right)$.

Let $C$ be the companion matrix of $(t-1) f(t)$. Then $C \in \mathrm{SL}_{n} \mathbb{Z}$, where $\operatorname{deg} f=n-1$. Consider the cyclic representation $\gamma: \pi \rightarrow \mathrm{SL}_{n} \mathbb{Z}$ sending each generator $x_{0}, x_{1}, \ldots, x_{k}$ of a Wirtinger presentation of $\pi$ to $C$. We have

$$
\tau_{\gamma}(t)=\frac{\operatorname{det} M_{\gamma \otimes \epsilon}^{0}}{\operatorname{det} \Phi\left(x_{0}-1\right)}=\frac{\operatorname{det} M_{\gamma \otimes \epsilon}^{0}}{f\left(t^{-1}\right)(t-1)} .
$$

The matrix $M_{\gamma \otimes \epsilon}^{0}$ can be obtained from the $(k \times k)$ Alexander matrix $M(t)$ by replacing each polynomial entry $\sum a_{i} t^{i}$ with the $(n \times n)$ block matrix $\sum a_{i}(t C)^{i}$. Since the $n \times n$ blocks commute,

$$
\operatorname{det} M_{\gamma \otimes \epsilon}^{0}=\prod_{\lambda} \operatorname{det} M(t \lambda),
$$

where $\lambda$ ranges over the eigenvalues of $C$, that is, the roots of $(t-1) f(t)$ (see Kovacs, Silver and Williams [9] for details). Hence

$$
\operatorname{det} M_{\gamma \otimes \epsilon}^{0}=\prod_{\lambda} \Delta(t \lambda)=\Delta(t) \prod_{\lambda: f(\lambda)=0} f(t \lambda) f\left(t^{-1} \lambda^{-1}\right) .
$$


Since $\Delta(t)$ and $\operatorname{det} M_{\gamma \otimes \epsilon}^{0}(t)$ are integral polynomials, so is

$$
g(t)=\prod_{\lambda: f(\lambda)=0} f(t \lambda) f\left(t^{-1} \lambda^{-1}\right) .
$$

Lemma 2.1 If $\operatorname{deg} f=2$, then $g(t)$ is reciprocal.

Proof Our assumptions about $f(t)$ imply that its roots have the form $\lambda,-\lambda^{-1}$, for some $\lambda \in \mathbb{C} \backslash\{0\}$. Then $g(t)=f(t \lambda) f\left(t^{-1} \lambda^{-1}\right) f\left(-t \lambda^{-1}\right) f\left(-t^{-1} \lambda\right)$ while $g\left(t^{-1}\right)=f\left(t^{-1} \lambda\right) f\left(t \lambda^{-1}\right) f\left(-t^{-1} \lambda^{-1}\right) f(-t \lambda)$. Observe that $g(t)$ and $g\left(t^{-1}\right)$ have the same roots:

- $f(t \lambda)$ and $f\left(-t^{-1} \lambda^{-1}\right)$ have roots: $t=1,-\lambda^{-2}$;

- $f\left(t^{-1} \lambda^{-1}\right)$ and $f(-t \lambda)$ have roots: $t=-1, \lambda^{-2}$;

- $f\left(-t \lambda^{-1}\right)$ and $f\left(t^{-1} \lambda\right)$ have roots: $t=1,-\lambda^{2}$;

- $f\left(-t^{-1} \lambda\right)$ and $f\left(t \lambda^{-1}\right)$ have roots: $t=-1, \lambda^{2}$.

It follows that $g\left(t^{-1}\right)=\alpha g(t)$, for some $\alpha \in \mathbb{C} \backslash\{0\}$. Letting $t=1$, we see that $\alpha=1$. Hence $g\left(t^{-1}\right)=g(t)$.

Remark 2.2 The numerator $\operatorname{det} M_{\gamma \otimes \epsilon}^{0}$ of Definition 1.1 is a polynomial invariant $D_{\gamma}(t)$ of $k$, well defined up to multiplication by units in $\mathbb{C}\left[t^{ \pm 1}\right]$ (see Silver and Williams [14]). Since $\Delta(t)$ is reciprocal, Lemma 2.1 implies that $D_{\gamma}(t)$ is reciprocal whenever $\operatorname{deg} f=2$. Example 2.5 below shows that this conclusion need not hold when $\operatorname{deg} f>2$.

Proposition 2.3 Let $f(t)$ be a polynomial as above with degree 2. If $f(t)$ is nonreciprocal, then $\tau_{\gamma}(t)$ is a non-reciprocal integral polynomial of the form $(t-1) h(t)$.

Proof From equation (2-1),

$$
\tau_{\gamma}(t)=\frac{f(t) f\left(t^{-1}\right) g(t)}{f\left(t^{-1}\right)(t-1)}=\frac{f(t) g(t)}{t-1} .
$$

Since $g(t)$ and $t-1$ are reciprocal but $f(t)$ is not, $\tau_{\gamma}(t)$ is non-reciprocal. To see that $\tau_{\gamma}(t)$ has the desired form, note that $(t-1)^{2}$ divides $g(t)$ since both factors $f(t \lambda), f\left(-t \lambda^{-1}\right)$ of $g(t)$ vanish when $t=1$. 
Example 2.4 Let $f(t)=t^{2}-t-1$. Then

$$
C=\left(\begin{array}{ccc}
0 & 0 & -1 \\
1 & 0 & 0 \\
0 & 1 & 2
\end{array}\right) .
$$

Computation shows that $g(t)=(t-1)^{2}(t+1)^{2}\left(t^{2}-3 t+1\right)\left(t^{2}+3 t+1\right)$. By equation $(2-2)$,

$$
\tau_{\gamma}(t)=\left(t^{2}-t-1\right)(t-1)(t+1)^{2}\left(t^{2}-3 t+1\right)\left(t^{2}+3 t+1\right),
$$

which is non-reciprocal.

Example 2.5 Let $f(t)=t^{3}-t-1$. Then

$$
C=\left(\begin{array}{cccc}
0 & 0 & 0 & -1 \\
1 & 0 & 0 & 0 \\
0 & 1 & 0 & 1 \\
0 & 0 & 1 & 1
\end{array}\right) .
$$

Computation shows that $g(t)=(t-1)^{3}\left(t^{3}-t-1\right)^{2}\left(t^{3}-t^{2}+2 t-1\right)\left(t^{6}+3 t^{5}+\right.$ $\left.5 t^{4}+5 t^{3}+5 t^{2}+3 t+1\right)$. The polynomial $f(t) f\left(t^{-1}\right) g(t)$ is the numerator $D_{\gamma}(t)$ of Wada's invariant (see Definition 1.1). It is non-reciprocal.

It is not difficult to see that for any cyclic representation, $D_{\gamma}(t)=\Delta_{\gamma}(t)$ (see [14, Section 3]) Hence this example shows that $\Delta_{\gamma}(t)$ can also be non-reciprocal.

\section{Sufficient condition for reciprocality}

If $\gamma: G \rightarrow \mathrm{GL}_{n} \mathbb{F}$ is a linear representation, then the $d u a l$ (or contragredient) representation $\bar{\gamma}$ is defined by

$$
\bar{\gamma}(g)={ }^{t} \gamma(g)^{-1}
$$

where ${ }^{t}$ denotes transpose.

The following elementary lemma is included for the reader's convenience.

Lemma 3.1 A representation $\gamma: G \rightarrow \mathrm{GL}_{n} \mathbb{F}$ is conjugate to its dual if and only if there exists a nondegenerate bilinear form $(v, w) \mapsto\{v, w\} \in \mathbb{F}$ on $V$ such that $\{v \cdot g, w \cdot g\}=\{v, w\}$ for all $v, w \in V$ and $g \in G$.

Proof Assume that $\bar{\gamma}$ is conjugate to $\gamma$. Then there exists a matrix $A \in \mathrm{GL}_{n} \mathbb{F}$ such that $A^{-1} \gamma(g) A={ }^{t} \gamma(g)^{-1}$, for all $g \in G$. Define $\{v, w\}=v A^{t} w$. Since $A$ is invertible, 
the bilinear form is nondegenerate. It is easy to check that $\{v \cdot g, w \cdot g\}=\{v, w\}$ for all $v, w \in V$.

Conversely, assume that $\gamma$ preserves a nondegenerate bilinear form $(v, w) \mapsto\{v, w\}$. There exists an invertible matrix $A \in \mathrm{GL}_{n} \mathbb{F}$ such that $\{v, w\}=v A^{t} w$. Since $\gamma$ preserves the form, we have $v \gamma(g) A^{t} \gamma(g){ }^{t} w=\{v \cdot g, w \cdot g\}=\{v, w\}=v A^{t} w$, for all $v, w \in V, g \in G$. It follows that $\gamma(g) A^{t} \gamma(g)=A$ for all $g \in G$. Hence $A^{-1} \gamma(g) A={ }^{t} \gamma(g)^{-1}$, and so $\bar{\gamma}$ is conjugate to $\gamma$.

As before, let $k$ be a knot with group $\pi$. Assume that $\gamma: \pi \rightarrow \mathrm{GL}_{n} \mathbb{F}$ is a representation, where $\mathbb{F}$ is an arbitrary field. As above, $V=\mathbb{F}^{n}$ is a right $\mathbb{Z}[\pi]$-module via $v \cdot g=$ $v \gamma(g)$, for all $v \in V$ and $\gamma \in \pi$. Let $W=\mathbb{F}^{n}$ with the dual $\mathbb{Z}[\pi]$-module structure given by $w \cdot g=w^{t} \gamma(t)^{-1}$.

Theorem 3.2 Assume that $\operatorname{det} M_{\gamma \otimes \epsilon}^{0} \neq 0$. If $\gamma$ is conjugate to its dual representation $\bar{\gamma}$, then both $\tau_{\gamma}(t)$ and $\Delta_{\gamma}(t)$ are reciprocal.

Proof The following argument is similar to those of Kitano [7] and of Kirk and Livingston [6].

Recall that $X$ is the exterior of $k$, endowed with a CW cell structure. Let $X^{\prime}$ be the same space but with the dual cell structure. Let $\bar{I}(t) \rightarrow \mathbb{F}(t)$ be the involution induced by $t \mapsto t^{-1}$.

Assume that $\gamma: \pi \rightarrow \mathrm{GL}_{n} \mathbb{F}$ is a representation that is conjugate to its dual. By Lemma 3.1 there exists a nondegenerate bilinear form $(v, w) \mapsto\{v, w\}$ such that $\{v \cdot g, w \cdot g\}=\{v, w\}$ for all $v, w \in V, g \in \pi$. Consider the twisted chain complexes

$$
\mathcal{C}_{*}=\left(\mathbb{F}(t) \otimes_{\mathbb{F}} V\right) \otimes_{\gamma} C_{*}(\tilde{X}), \mathcal{D}_{*}=\left(\mathbb{F}(t) \otimes_{\mathbb{F}} W\right) \otimes_{\bar{\gamma}} C_{*}\left(\tilde{X}^{\prime}, \partial \tilde{X}^{\prime}\right),
$$

where $\tilde{X}$ and $\tilde{X}^{\prime}$ denote universal covering spaces of $X$ and $X^{\prime}$, respectively. We abbreviate these by $V_{\gamma \otimes \epsilon} \otimes C_{*}(\tilde{X})$ and $V_{\bar{\gamma} \otimes \epsilon} \otimes C_{*}(\tilde{X})$, respectively.

Define a bilinear pairing $\mathcal{C}_{q} \times \mathcal{D}_{3-q} \rightarrow \mathbb{F}(t)$ by

$$
\left\langle p \otimes v \otimes z_{1}, q \otimes w \otimes z_{2}\right\rangle=\sum_{g \in \pi}\left(z_{1} \cdot g z_{2}\right) p \bar{q}\{v \cdot g, w\},
$$

where $z_{1} \cdot g z_{2}$ is the algebraic intersection number of cells $z_{1}$ and $g z_{2}$. We extend linearly.

The pairing induces a $\mathbb{F}(t)$-module isomorphism $\mathcal{D}_{3-q} \rightarrow \overline{\operatorname{Hom}}\left(\mathcal{C}_{q}, \mathbb{F}(t)\right)$, where $\overline{\text { Hom }}$ denotes the dual space with $(q \cdot h)(z)=\bar{q}(h(z))$, for all $q \in \mathbb{F}(t), z \in \mathcal{C}_{q}$. Consequently, 
there exists a nondegenerate pairing $H_{q}(X ; V(t)) \times H_{3-q}\left(S^{\prime}, \partial X^{\prime} ; W(t)\right) \rightarrow \mathbb{F}(t)$. Since the torsion of $\mathcal{C}_{*}$ is defined, by our hypothesis, the torsion of $\mathcal{D}_{*}$ is too.

Choose a basis $\left\{v_{i}\right\}$ over $\mathbb{F}$ for $V$ and lifts to $\tilde{X}$ of simplices of $X$ to get a preferred $\mathbb{F}(t)$-basis for $\mathcal{C}_{*}$. Basis elements have the form $1 \otimes v_{i} \otimes z_{j}$. Then $\mathcal{D}_{*}$ has a natural dual basis over $\mathbb{F}(t)$ obtained by picking a basis for $W$ that is dual to the basis for $V$ with respect to $\{$,$\} , and dual cells in \tilde{X}^{\prime}$ of the fixed lifts of simplices of $X$. As observed by Kirk and Livingston [6], the bases for $\mathcal{C}_{*}$ and $\mathcal{D}_{*}$ that we build are dual with respect to bilinear form (3-1).

Let $\tau\left(X ; V_{\gamma \otimes \epsilon}\right)$ denote the torsion of $\mathcal{C}_{*}$. Similarly, let $\tau\left(X^{\prime}, \partial X^{\prime} ; V_{\bar{\gamma} \otimes \epsilon}\right)$ denote the torsion of $\mathcal{D}_{*}$. Then $\tau\left(X ; V_{\gamma \otimes \epsilon}\right)=\tau\left(X^{\prime}, \partial X^{\prime} ; V_{\bar{\gamma} \otimes \bar{\epsilon}}\right)$ by Milnor [12, Theorem 1']. Futhermore,

$$
\begin{array}{rlr}
\tau\left(X^{\prime}, \partial X^{\prime} ; V_{\bar{\gamma} \otimes \bar{\epsilon}}\right) & =\tau\left(X, \partial X ; V_{\bar{\gamma} \otimes \bar{\epsilon}}\right) & \text { (by subdivision) } \\
& =\tau\left(X, \partial X ; V_{\gamma \otimes \bar{\epsilon}}\right) & \text { (since } \gamma \text { is conjugate to } \bar{\gamma}) \\
& =\bar{\tau}\left(X, \partial X ; V_{\gamma \otimes \epsilon}\right) \\
& =\bar{\tau}\left(X ; V_{\gamma \otimes \epsilon}\right),
\end{array}
$$

using Milnor [11, Lemma 2] and $\tau\left(\partial X ; V_{\gamma \otimes \epsilon}\right)=1$ (see Kirk and Livingston [6]). Hence

$$
\tau_{\gamma}(t)=\tau\left(X ; V_{\gamma \otimes \epsilon}\right)=\bar{\tau}\left(X ; V_{\gamma \otimes \epsilon}\right)=\bar{\tau}_{\gamma}(t) .
$$

In order to show that $\Delta_{\gamma}(t)$ is also reciprocal, we need the fact from [6] that $\Delta_{\gamma}(t)$ is equal to $\tau_{\gamma}(t)$ times the 0 th elementary divisor of $H_{0}\left(X ; V\left[t^{ \pm 1}\right]\right)$, computed using the chain complex (1-2). We observe that $H_{0}\left(X ; V\left[t^{ \pm 1}\right]\right)$ is the cokernel of the boundary homomorphism $\partial_{1}$. For any $g \in \pi$, the set of eigenvalues of $\gamma(g)$ is closed under inversion, since $\gamma$ is conjugate to its dual. It follows that the 0th elementary of $H_{0}\left(X ; V\left[t^{ \pm 1}\right]\right)$ is reciprocal. Hence so is $\Delta_{\gamma}(t)$.

Remark 3.3 If $\mathbb{F}=\mathbb{R}$, and the bilinear form in Lemma 3.1 is positive-definite, then by considering a basis for $V$ that is orthonormal with respect to the form, we see that $A$ is the identity matrix. In this case, $\gamma(g)={ }^{t} \gamma(g)^{-1}$ for all $g \in G$, and hence $\gamma$ is conjugate to an orthogonal representation. Similarly, if $\mathbb{F}=\mathbb{C}$ and the bilinear form is hermitian and positive-definite, $\gamma$ is conjugate to a unitary representation.

Corollary 3.4 If $\gamma: \pi \rightarrow \mathrm{Sp}_{2 n} \mathbb{F}$ is a symplectic representation, then $\tau_{\gamma}(t)$ is reciprocal.

Proof The representation preserves the bilinear form given by $A=\left(\begin{array}{cc}0_{n} & I_{n} \\ -I_{n} & 0_{n}\end{array}\right)$. Since $\mathrm{Sp}_{2} \mathbb{F}=\mathrm{SL}_{2} \mathbb{F}$, the following is immediate. 
Corollary 3.5 If $\gamma$ is any representation of $\pi$ in $\mathrm{SL}_{2} \mathbb{F}$, then $\tau_{\gamma}(t)$ is reciprocal.

Corollary 3.5 shows that Example 2.4 is, in a sense, the simplest possible.

Acknowledgments The authors thank Kunio Murasugi, Stefan Friedl and the referee for their insights and helpful suggestions. We are grateful to Walter Neumann for suggesting the possibility that a representation that is not conjugate to its dual might yield non-reciprocal torsion.

The second and third authors were partially supported by NSF grant DMS-0706798.

\section{References}

[1] J C Cha, Fibred knots and twisted Alexander invariants, Trans. Amer. Math. Soc. 355 (2003) 4187-4200 MR1990582

[2] S Friedl, S Vidussi, Twisted Alexander polynomials and symplectic structures, Amer. J. Math. 130 (2008) 455-484 MR2405164

[3] S Friedl, S Vidussi, Twisted Alexander polynomials, symplectic 4-manifolds and surfaces of minimal complexity, from: "Algebraic topology - old and new", Banach Center Publ. 85, Polish Acad. Sci. Inst. Math., Warsaw (2009) 43-57 MR2503517

[4] J A Hillman, C Livingston, S Naik, Twisted Alexander polynomials of periodic knots, Algebr. Geom. Topol. 6 (2006) 145-169 MR2199457

[5] A Kawauchi, A survey of knot theory, Birkhäuser Verlag, Basel (1996) MR1417494 Translated and revised from the 1990 Japanese original by the author

[6] P Kirk, C Livingston, Twisted Alexander invariants, Reidemeister torsion, and CassonGordon invariants, Topology 38 (1999) 635-661 MR1670420

[7] T Kitano, Twisted Alexander polynomial and Reidemeister torsion, Pacific J. Math. 174 (1996) 431-442 MR1405595

[8] T Kitayama, Refinement of twisted Alexander invarariants and sign-determined Reidemeister torsions arXiv:0705.2371

[9] I Kovacs, D S Silver, S G Williams, Determinants of commuting-block matrices, Amer. Math. Monthly 106 (1999) 950-952 MR1732497

[10] X S Lin, Representations of knot groups and twisted Alexander polynomials, Acta Math. Sin. (Engl. Ser.) 17 (2001) 361-380 MR1852950

[11] J Milnor, Two complexes which are homeomorphic but combinatorially distinct, Ann. of Math. (2) 74 (1961) 575-590 MR0133127

[12] J Milnor, A duality theorem for Reidemeister torsion, Ann. of Math. (2) 76 (1962) 137-147 MR0141115 
[13] D Rolfsen, Knots and links, Mathematics Lecture Series 7, Publish or Perish, Berkeley, CA (1976) MR0515288

[14] D S Silver, S G Williams, Dynamics of twisted Alexander invariants, Topology Appl. 156 (2009) 2795-2811 MR2556037

[15] G Torres, R H Fox, Dual presentations of the group of a knot, Ann. of Math. (2) 59 (1954) 211-218 MR0062439

[16] M Wada, Twisted Alexander polynomial for finitely presentable groups, Topology 33 (1994) 241-256 MR1273784

JAH: School of Mathematics and Statistics F07, University of Sydney Sydney, NSW 2006, Australia

DSS, SGW: Department of Mathematics and Statistics, University of South Alabama Mobile AL 36688, USA

jonathan.hillman@sydney.edu.au, silver@jaguar1.usouthal.edu, swilliam@jaguar1.usouthal.edu

http://www.maths.usyd.edu.au/u/jonh/, http://www.southalabama.edu/mathstat/personal_pages/silver/, http://www.southalabama.edu/mathstat/personal_pages/williams/

Received: 18 June 2009 